\title{
CLOUDINESS IMAGES MULTILEVEL SEGMENTATION BY PIECEWISE LINEAR APPROXIMATION OF CUMULATIVE HISTOGRAM
}

\author{
Roman Melnyk, Ruslan Tushnytskyy, Roman Kvit
}

\author{
Lviv Polytechnic National University, Ukraine \\ ramelnyk@polynet.lviv.ua, ruslan.tushnytskyy@gmail.com,roman.i.kvit@1pnu.ua
}

Paper history:

Received 11 October 2019

Received in revised form 3 February 2020

Accepted 2 March 2020

Available online 14 June 2020
Keywords:

approximation;

threshold;

piecewise-linear function;

cumulative histogram.

\begin{abstract}
The Ramer-Douglas-Peucker algorithm for piecewise approximation is used for image multilevel segmentation. The cumulative histogram is selected as a function for approximation. The algorithm allows you to determine threshold values of continuous and discrete images. The algorithm is used to separate cloudiness from background and to calculate cloudiness intensity. The found points of the approximated function have been accepted to change pixel intensity by proposed formulas. The algorithm efficiency is compared with those based on ordinary and cumulative histograms. By controlling the number of points for piecewise linear approximation function, the necessary segmentation accuracy can be achieved. The algorithm complexity is linear to the number of image pixels and to the number of intensity steps. The developed algorithm is applied to the satellite map images to separate clouds of different intensity. The extracted clouds of different intensity are used to classify regions by cloudiness with a developed clustering algorithm. Testing and experimental results are presented.
\end{abstract}

Copyright (C) Research Institute for Intelligent Computer Systems, 2020. All rights reserved.

\section{INTRODUCTION}

Determination of the image features requires fast algorithms of image segmentation. Nowadays, there is a great variety of publications on methods for image segmentation. They can be generally divided into two classes: those that are based on finding the intensity threshold and those that divide the image into regions having certain features. The first ones determine the intensity thresholds and are based on histograms. Among them, the algorithms of determining the minimal intensity [1], convexity [2], moments [3], entropy [4], minimal errors [5, 6], etc. can be distinguished. The typical example of the methods from the second class is the graph-based image segmentation [7]. In the paper [8] the improved Otsu method that constrains the search interval of gray levels is proposed. In the work [9] the required threshold is determined in the receiver operating characteristic space.

Multilevel thresholding is of great importance. However, the computational complexity of it in many algorithms increases exponentially with the number of thresholds. So, authors try to minimize the algorithm complexity. In the paper [10] a criterion for maximizing a modified between-class variance is proposed and a recursive algorithm is designed to efficiently find the optimal threshold using stored in a look-up table. The publication [11] proposes new method based on quantum particle swarm optimization (QPSO) algorithm. In the work [12] starting from the extreme pixel values at both ends of the histogram plot, the algorithm is applied recursively on sub-ranges computed from the previous step, so as to find a threshold level and a new sub-range for the next step, until no significant improvement in image quality can be achieved.

The paper [13] proposes multilevel image thresholding for image segmentation using several recently presented P-metaheuristic algorithms, including whale optimization algorithm, grey wolf optimizer (GWO), teaching-learning-based optimization algorithm and some others.

The paper [14] proposes a method by using cluster organization from the histogram of an image. A new similarity measure proposed is based on inter-class variance of the clusters to be merged and the intra-class variance of the new merged cluster. 
A new method of multilevel thresholds for image segmentation using grey wolf optimizer is proposed [15]. This metaheuristic algorithm is applied to multilevel threshold problem using Kapur's entropy and Otsu's between class variance functions.

The drawbacks of the abovementioned and some other algorithms are the different thresholds for similar images even within the algorithms of the same class. Most algorithms are fairly bulky, especially those using graph models or those based on statistical calculations. Modern CBIR-systems process millions of images in real time and therefore need extremely fast and quite accurate image feature determination tools. Segmentation algorithms are an important part of these tools.

In this paper we present very fast and simple algorithm having a linear algorithmic complexity and clear physical explanation. It needs to calculate the image cumulative histogram and to find the extreme coordinate in the interval $0-255$ as many times as many levels of segmentation are needed.

Comparatively with the work [16], where cloudiness was considered as one object for analysis, in this paper cloudiness is divided into three objects: low, middle and high clouds.

The paper is based on the research provided by the grant support of the State Fund for Fundamental Research (project No 33651).

\section{PIECEWISE-LINEAR APPROXIMATION}

We want to approximate a function $f(x)$ by piecewise-linear function $g(x)$ determined on the interval $a_{i} \leq x_{i} \leq a_{i+1}$ with a set of sections having the end points $\left(\alpha_{i}, \beta_{i}\right)$ and $\left(\alpha_{i+1}, \beta_{i+1}\right)$. The function $g(x)$ minimizes the approximation error $\varepsilon$ got by the following formula:

$$
\varepsilon=\min _{\alpha_{i} \leq x \leq \alpha_{i+1}}|g(x)-f(x)|
$$

The convex function $f(x)$ can be approximated by the piecewise-linear function $g(x)$ having $N$ segments and determined on the interval $a_{0} \leq x \leq a_{N}$ with a set of points (nodes) $\left(\alpha_{i} \beta_{i}\right)_{i=0}^{N}$ connected these $N$ segments.

The approximation function is presented as:

$$
g(x)=\sum_{i=0}^{N-1} g_{i}(x),
$$

where

$$
g_{i}(x)= \begin{cases}m_{i} x+b_{i} & \text { for } a_{i} \leq x \leq a_{i+1}, \\ 0 & \text { in other case, }\end{cases}
$$

$$
\begin{gathered}
m_{i}=\frac{\beta_{i+1}-\beta_{i}}{\alpha_{i+1}-\alpha_{i}} \\
b_{i}=\beta_{i}-m_{i} \alpha_{i} \text { for } i=\overline{0, N-1} .
\end{gathered}
$$

For piecewise-linear approximation of the cumulative histogram function the Ramer-DouglasPeucker (RDP) algorithm [17, 18] was taken as a basic one.

The essence of the algorithm is approximating initial curve $f_{0}$ represented by the set of points $P_{i}\left(x_{i}, y_{i}\right) \in P, i=\overline{1, n}$, with piecewise-linear function $f_{a}$ represented with points $O_{j}\left(x_{j}, y_{j}\right) \in O, j=\overrightarrow{1, m}$, $m \leq n$.

The algorithm defines maximum distance (tolerance) between the original and the approximating function:

$$
t=\max \left|f_{0}\left[P_{i}\left(x_{i}, y_{i}\right)\right]-f_{a}\left[O_{i}\left(x_{i}, y_{i}\right)\right]\right|, i=\overline{1, n} .
$$

This distance must be less than assigned maximum allowable tolerance, which determines desired accuracy of the approximation.

The initial curve is an ordered set of points or lines within $\varepsilon>0$. The process of the curve approximation is shown in Fig. 1.

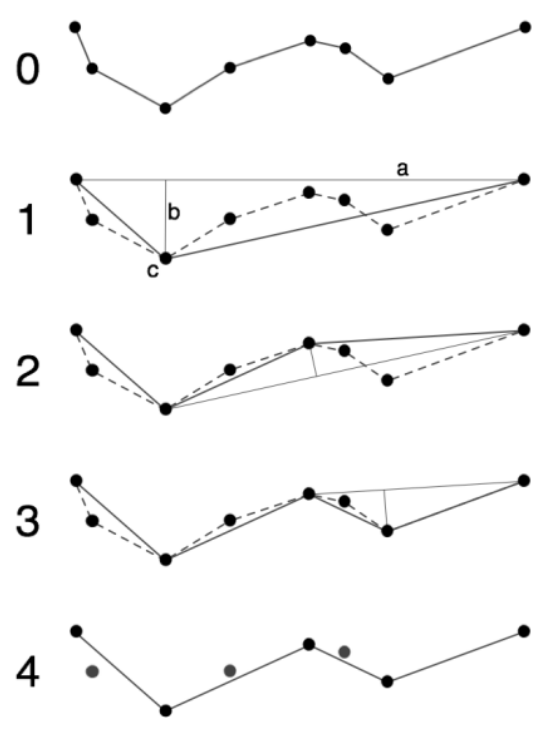

Figure 1 - Steps of curve approximation by RDP algorithm

Input data for the algorithm are the coordinates of all points of a function including the first and the last ones. The algorithm recursively divides the line. The first and the last points are kept unchanged. Then the algorithm finds the point farthest from the line drawn through the first and the last.

If the point is located at distance less than $\varepsilon$, then all the points that have not yet been signed for 
storing may be removed from the set and received line will smooth curve with an accuracy not less than $\varepsilon$.

If the distance is more than $\varepsilon$, then the algorithm recursively calls itself on the set of points from first to the current and from the current to the endpoint (which means that current point will be signed for storing).

After all the recursive calls output polygon is based only on those points that were signed for storing.

The results of the cumulative histogram function approximation using RDP algorithm for the test image for some value of the maximum allowable deviation (tolerance) are presented in Fig. 2.

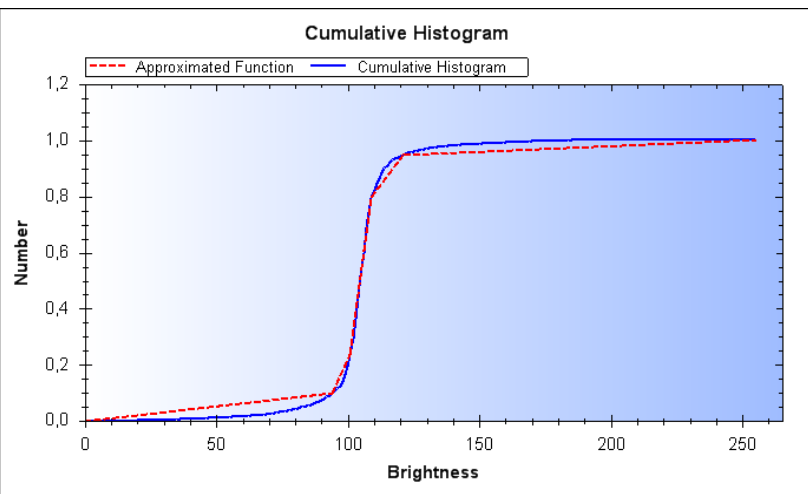

Figure 2 - Cumulative histogram approximation by RDP algorithm

To estimate a quality of segmented and transformed images we use the root mean-squared error (MSE) and peak signal-to-noise ratio (PSNR):

$$
\begin{aligned}
& M S E=\sqrt{\frac{\sum_{M, N}\left[I_{1}(m, n)-I_{2}(m, n)\right]^{2}}{M * N},} \\
& P S N R=20 \lg \left(\frac{255}{M S E}\right) .
\end{aligned}
$$

Here $I_{1}(m, n)$ and $I_{2}(m, n)$ are the original and transformed images, of size $M \times N$, respectively.

After applying the present algorithm recursively a few times, PSNR of the segmented image is found to saturate. This property can be used to obtain the appropriate number of thresholds and to select the best quality of approimation from formulas (13)(16).

Computation complexity of the approximation algorithm could be determined by a number of thresholds.

In the case, when its number is $k$, a complexity of the approximation algorithm is linear to a number of pixels and equals $O(N \times M+256 \times k)$.

\section{MULTILEVEL THRESHHOLDS FOR IMAGE SEGMENTATION}

For the image histogram

$$
V=\sum_{i=1}^{n} V(i)
$$

it is easy to calculate the cumulative histogram

$$
V_{F}(s)=\sum_{i=1}^{s} V(i)
$$

where $V$ is the overall number of image pixels, $V(i)$ is intensity frequencies, $V_{F}(s)$ is the accumulating frequency for the given intensity, $n$ is the number of cumulative histogram intervals, $s$ and $i$ are interval numbers (intensity values).

We apply the RDP algorithm to the function $V_{F}(s)$ to get the main threshold value as well as other possible values for multilevel segmentation.

To confirm or to deny robustness of the proposed approach we use two "model" algorithms: known Otsu algorithm [1] and our own approach [19]. We consider two types of images: having discrete steps of intensity and having a "continuous" type with the intensity step as 1 .

In Fig. 3a, an example of the "pyramid" image is shown. In Fig. 3b, we see its cumulative histogram and two-lines approximating function got by the RDP algorithm. The middle point indicates the main threshold value $h=51$ for the "pyramid" image. The same value demonstrates the Otsu algorithm.

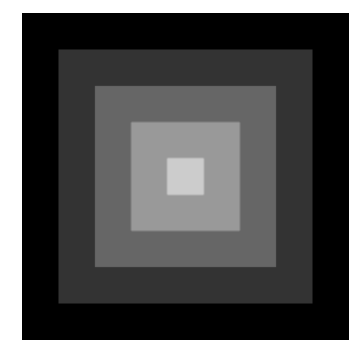

a)

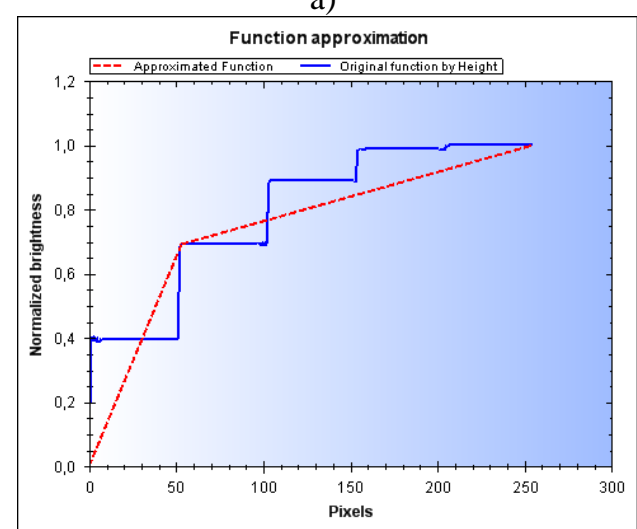

b)

Figure 3 - "Pyramid" image (a), its cumulative histogram (blue lines) and approximating function (red lines) by the RDP algorithm (b)

The lower and the upper parts of segmented image are shown in Fig. 4. 


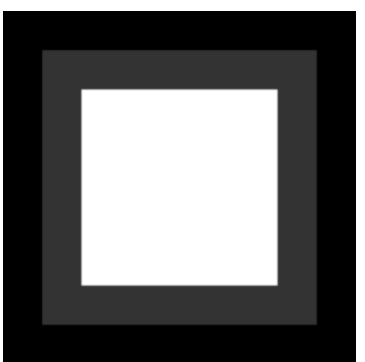

a)

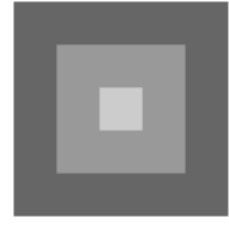

b)
Figure 4 - Lower (a) and upper (b) parts of "pyramid" image

If we apply the Otsu algorithm for both parts of the image, we will have the following threshold values: $h\left(O_{1}\right)=67, h\left(O_{u}\right)=163$. By reducing the tolerance, the approximation algorithm consequently gives all possible threshold values (Fig. 5 and Table 1). They do not contain those points received from the Otsu algorithm.

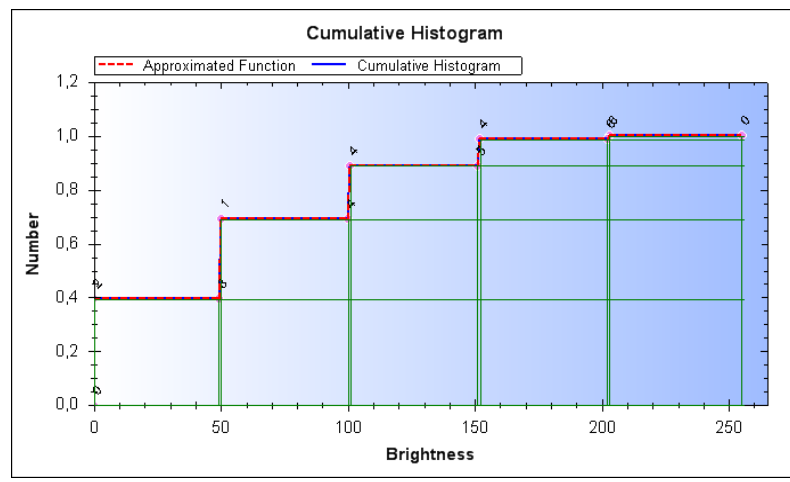

Figure 5 - Function approximation by the RDP algorithm

The final version of the approximating function has 11 points: two outside and nine inner points. Tolerances and a number of points in the piecewise linear approximating function are given in Table 1.

Table 1. Number of approximating points by tolerance

\begin{tabular}{|c|c|}
\hline Tolerance & $\begin{array}{l}\text { Number } \\
\text { of points }\end{array}$ \\
\hline $0,496-1,000$ & 2 \\
\hline $0,396-0,495$ & 3 \\
\hline $0,291-0,395$ & 4 \\
\hline $0,143-0,290$ & 5 \\
\hline $0,097-0,142$ & 8 \\
\hline $0,007-0,096$ & 9 \\
\hline $0,001-0,006$ & 11 \\
\hline
\end{tabular}

Values of the approximating function, all threshold values and an order of its determination are given in Table 2 .

Table 2. Approximating function

\begin{tabular}{|c|c|c|}
\hline Intensity & Function & $\begin{array}{c}\text { Order of } \\
\text { threshold }\end{array}$ \\
\hline 0 & 0,395 & 2 \\
\hline 49 & 0,395 & 3 \\
\hline 50 & 0,691 & 1 \\
\hline 100 & 0,691 & 4 \\
\hline 101 & 0,888 & 4 \\
\hline 151 & 0,888 & 5 \\
\hline 152 & 0,987 & 4 \\
\hline 202 & 0,987 & 6 \\
\hline 203 & 1,000 & 6 \\
\hline 255 & 1,000 & 0 \\
\hline
\end{tabular}

For the "pyramid" example we have 5 levels of pixels. If we want to continue to divide pixels by assigning additional colors, we should divide pixels from any of given levels.

For the next test we have chosen the wide known image of "12". The image and its cumulative histogram are shown in Fig. 6.

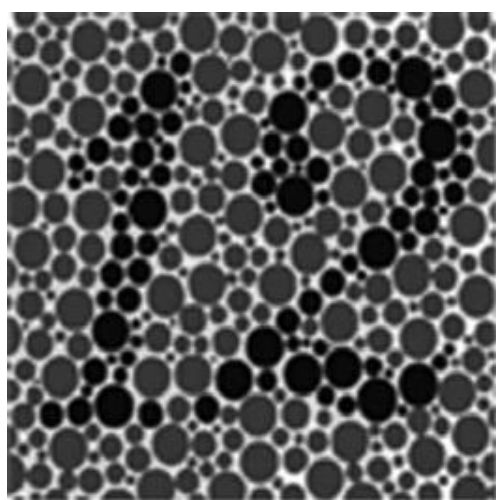

a)

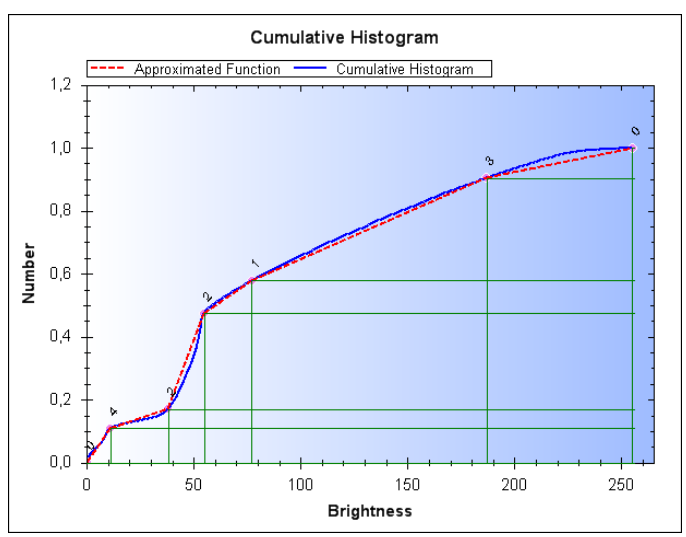

b)

Figure 6 - "12" image (a) and its cumulative histogram by a blue line and approximation function by a red line having seven levels (b)

In Fig. 6b, the cumulative histogram is approximated by the RDP algorithm. The first received point indicates the threshold value $h t=77$. The Otsu algorithm gives $h t=107$. The approximating function has 7 points. Tolerances and a number of points in the piecewise linear approximating function are given in Table 3 .

Table 3. Number of approximating points by tolerance 


\begin{tabular}{|c|c|}
\hline Tolerance & $\begin{array}{c}\text { Number } \\
\text { of points }\end{array}$ \\
\hline $0,277-1,000$ & 2 \\
\hline $0,116-0,276$ & 3 \\
\hline $0,065-0,115$ & 4 \\
\hline $0,060-0,064$ & 5 \\
\hline $0,045-0,059$ & 6 \\
\hline $0,035-0,044$ & 7 \\
\hline$\ldots \ldots \ldots \ldots \ldots . .$. & $\ldots$. \\
\hline $0,000-0,001$ & 40 \\
\hline
\end{tabular}

Values of the approximating function for seven points, their threshold values and an order of determination are given in Table 4 . We can observe these points in Fig. 6.

Table 4. Approximating function

\begin{tabular}{|c|c|c|}
\hline Intensity & Function & $\begin{array}{l}\text { Order of } \\
\text { threshold }\end{array}$ \\
\hline 12 & 0,108 & 4 \\
\hline 38 & 0,169 & 5 \\
\hline 55 & 0,473 & 2 \\
\hline 77 & 0,578 & 1 \\
\hline 187 & 0,903 & 3 \\
\hline 255 & 1,000 & 0 \\
\hline
\end{tabular}

The approximating algorithm gives maximal number of points 40 and their placement we can see in Fig. 7.

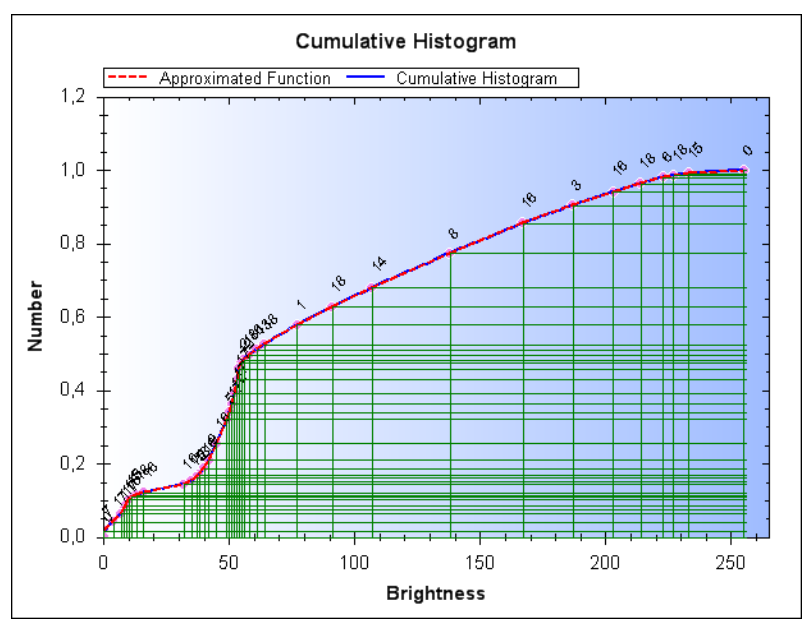

Figure 7 - Cumulative histogram of "12" image and approximating function by forty points

It is difficult to determine one of the found points $(h t=12)$ by known algorithms. The low segment for this point is shown in Fig. 8.

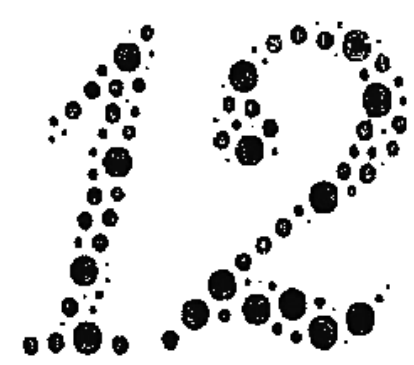

Figure 8 - "12" image segmented with threshold value

$$
h=12
$$

For comparison with the other approach we use a concept of a hypothetical image, a set of pixels, in which all the intensities are represented by the same number of pixels. The number of pixels for each intensity value is $N \times M / n$, where $N, M$ are the sizes of the image for which the threshold segmentation is being searched, $n$ is a number of cumulative histogram intervals.

For a hypothetical image, a normalized cumulative histogram is constructed according to the following formula:

$$
V_{F H}(s)=s / n, \quad s=\overline{1, n},
$$

where $V_{F H}(s)$ is a number of pixels (accumulated frequency) of the hypothetical image within the intensity interval $1 \div s$.

We construct a function $D(s)$ of the difference between the cumulative histograms of real and hypothetical images:

$$
D(s)=V_{F}(s)-V_{F H}(s), \quad s=\overline{1, n} .
$$

In Fig. 9, the plot of the $D(s)$ function, the difference between the cumulative histograms of the "12" image real and hypothetical images, is given.

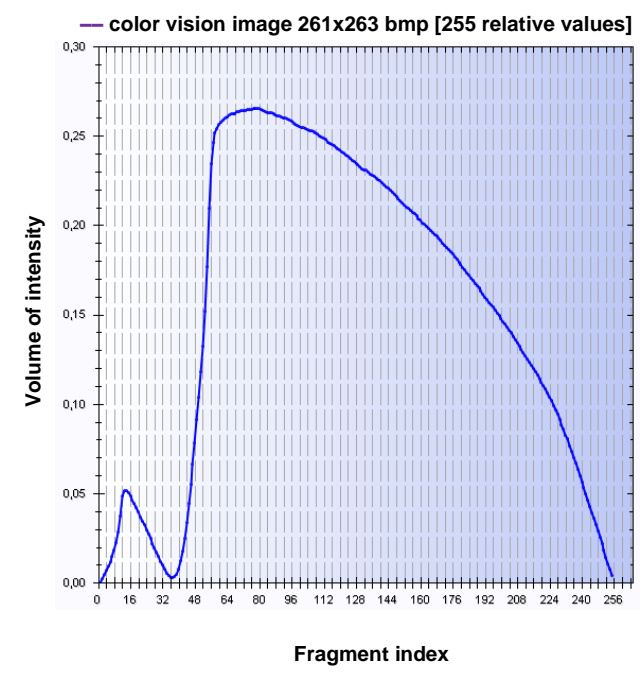

Figure 9-Function $D(s)$ for the "12" image 
The function $D(s)$ indicates the intervals in which the frequencies of image pixels are larger or smaller than the corresponding values of the hypothetical image, when they are increasing or decreasing. The function is characterized by the special points: extreme, inflection points or fracture.

In particular, in Fig. 9, we can see that the $D(s)$ function has three extremes. We consider that the coordinates of the extremes indicate possible thresholds for the image segmentation. In Fig. 9, three extremes points have the following threshold values: $h=12, h=38$ and $h=77$. They coincide with first, second and forth points from Table 4. As we can see there are no other extreme points on the $D(s)$ plot. So, the next threshold values can't be discovered. But the approximation algorithm allows us to find all possible threshold values.

\section{MULTILEVEL SATELLITE MAP IMAGE SEGMENTATION}

For cloudiness monitoring in different regions of the Earth surface the satellite map images are widely used. The popular services from free resources [2022] give the map images ordered from different parts of the world. So, the satellite maps of Ukraine with cloudiness are being obtained and stored. For every interval of cloudiness, we need to calculate intensity, mass, moving direction and other cloud properties. To get an access to the corresponding cloud areas the developed algorithm was applied to satellite image in Fig. 10.

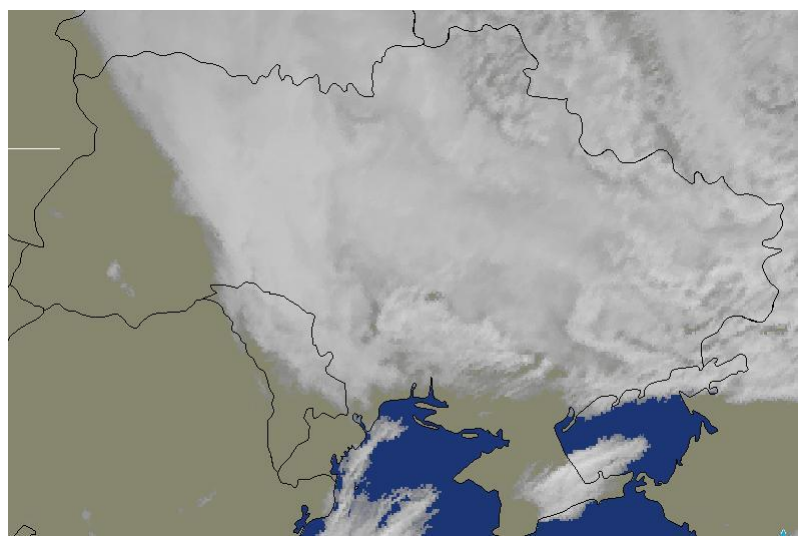

Figure 10 - Satellite map image

To the original image in Fig. 10, the approximation algorithm was applied.

In Fig. 11, we see how the cumulative histogram is approximated by piecewise the linear function with 12 intervals.

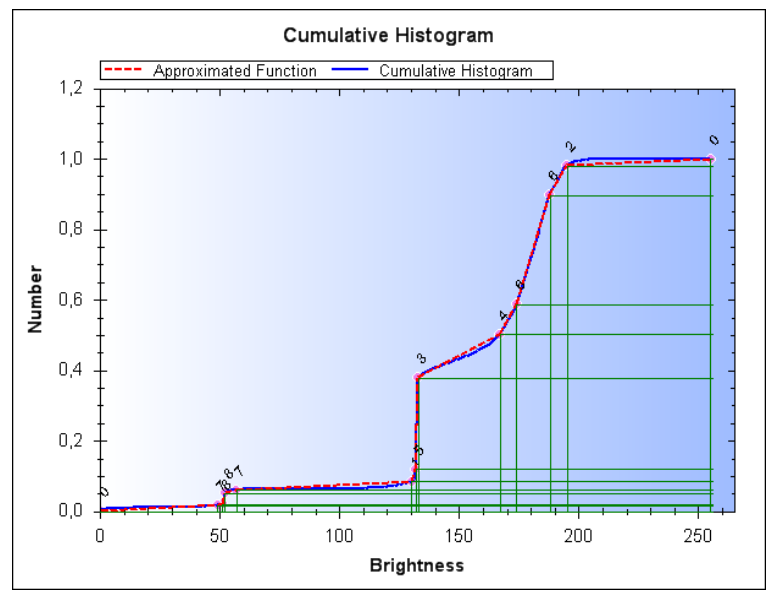

Figure 11 - Cumulative histogram of map image and approximating function by seven points

Values of the approximating function for six points, their threshold values and an order of determination are given in Table 5. We can observe these points in Fig. 13.

Table 5. Approximation function

\begin{tabular}{|c|c|c|}
\hline Intensity & Function & $\begin{array}{c}\text { Order of } \\
\text { threshold }\end{array}$ \\
\hline 130 & 0,084951 & 1 \\
\hline 132 & 0,120179 & 5 \\
\hline 133 & 0,379789 & 3 \\
\hline 167 & 0,501882 & 4 \\
\hline 195 & 0,980981 & 2 \\
\hline 255 & 1,000000 & 0 \\
\hline
\end{tabular}

The final version of the approximating function has 12 points: two external and ten internal. The coordinates of intensity intervals in the piecewise linear function give us four opportunities to change a color in the interval: left or right intensity coordinates, center or weighted mean intensity value of all pixels in the interval. So, we can choose one from four formulas:

$$
\begin{gathered}
I_{s k}(i, j)=I_{k}(l), \quad(i, j) \in P_{k}, \\
I_{s k}(i, j)=I_{k}(r), \quad(i, j) \in P_{k}, \\
I_{s k}(i, j)=\left[I_{k}(l)+I_{k}(r)\right] / 2, \quad(i, j) \in P_{k},
\end{gathered}
$$

$$
I_{s k}(i, j)=\frac{1}{n_{k}} \sum_{z=1}^{n_{k}} n_{k z} I_{k z}, \quad(i, j) \in P_{k}
$$

Here $k$ is an index of the intensity interval, $n_{k}$ is a number of pixels in the k-th interval, $I_{s k}(i, j)$ denotes pixels of $k$-th interval of the new 
segmented image, $l$ and $r$ indicate its left and right borders, $n_{k z}$ is a number of pixels having intensity $I_{k z}$ in $k$-th interval, $P_{k}$ is a set of pixels belonging to $I_{k}$ i.e. the $k$-th interval.

The main threshold value given by the approximating algorithm is $h=130$. By Otsu $h=145$ and some clouds are cut. Our threshold cuts an image segment containing the Black and Azov Seas. The next three thresholds divide cloudiness into three intervals: light dark $(133-167)$, light
(167-195) and light white (195-255). In Fig. 12, these three types of cloudiness are shown. To exclude influence of other colors they are marked as black.

Black color allows us to perform clustering of the map and to evaluate intensity of cloudiness in different regions of the territory. In Fig. 12, an example of the clustered map and corresponding dendogram are given.

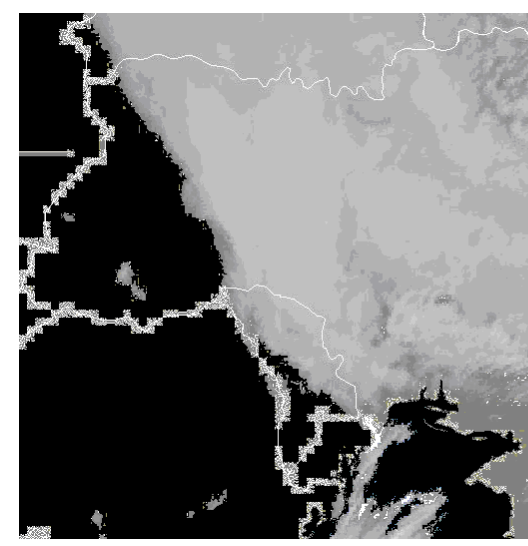

a)
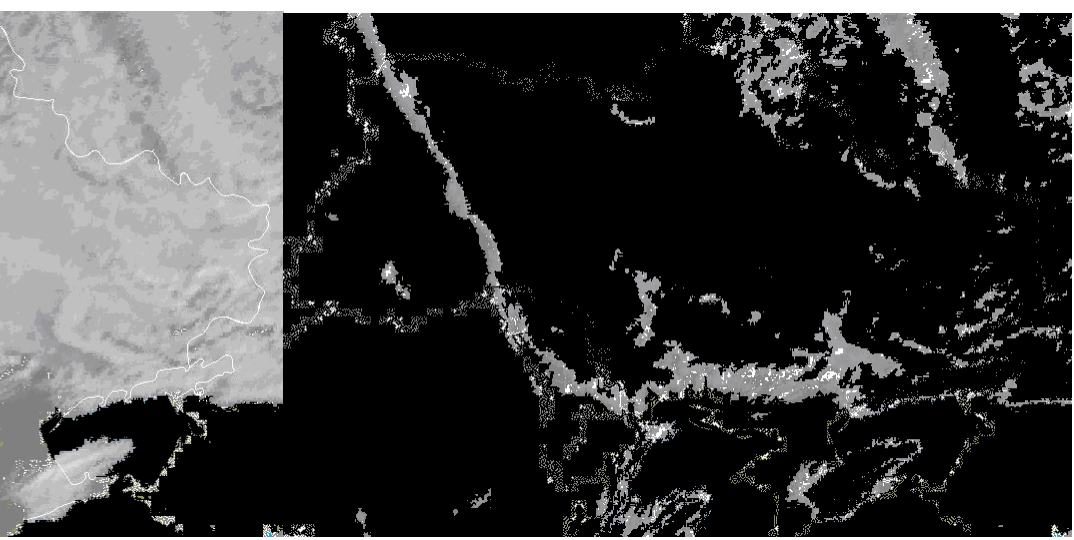

b)

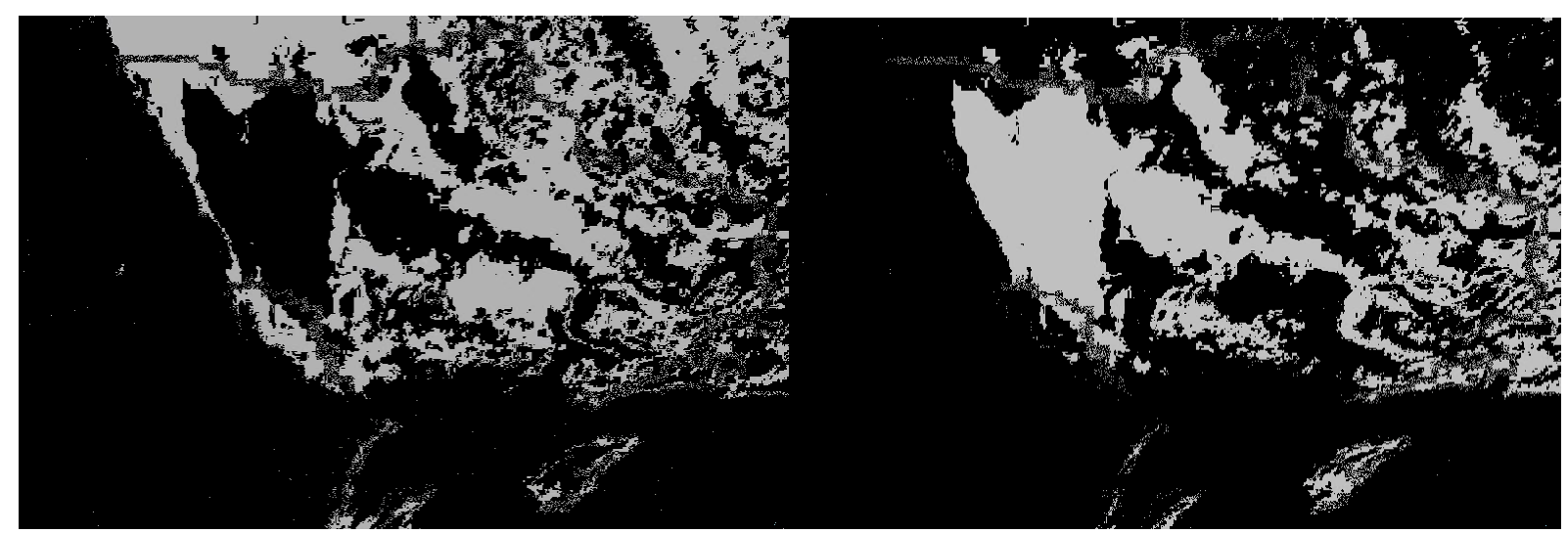

c)

d)

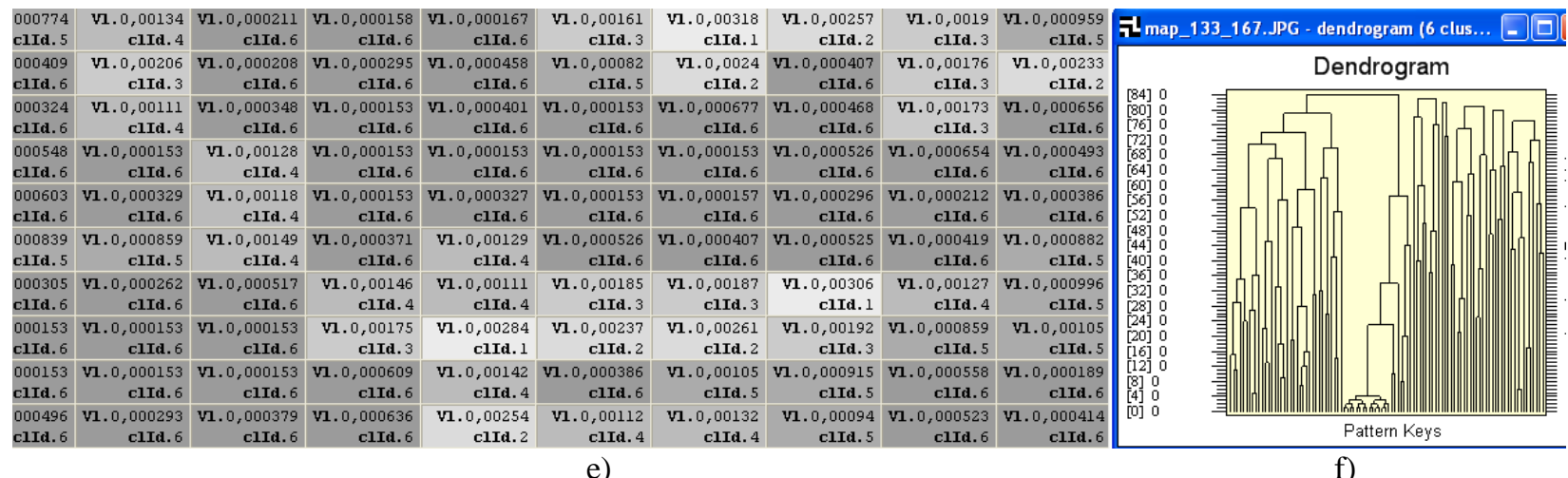

e)

Figure 12 - Segmented images of satellite map: (a) 130-255, (b) 133-167, (c) 167-195, (d) 195-255, (e) - clustering matrix for (b), (f) - dendogram 
The next experiment was held with multilevel segmentation. The coordinates of intervals in the piecewise linear function give us four opportunities to change a color in the interval: left or right coordinates, center or weighted mean values. Experiments show the smallest error for the last case. So, in Fig. 13, we have original satellite map image and approximated images for different numbers of approximation levels: 4, 6, 8, 10 as well as their cumulative histograms.

The performance metrics for checking the effectiveness of the method are chosen as computational time and PSNR which is used to determine the quality of the segmented image. PSNR values for different types of changing the intensity pixel exchange in the determined intensity intervals are given in Table 6 .

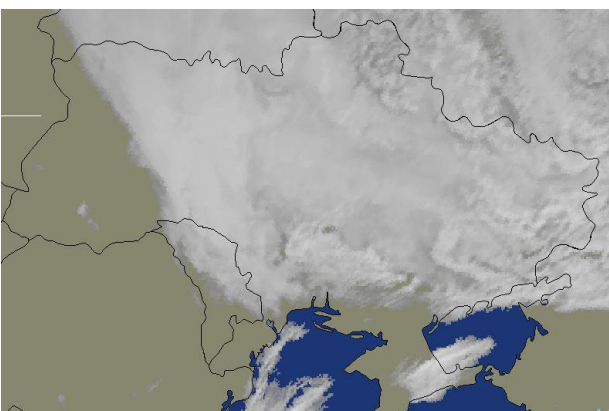

a)

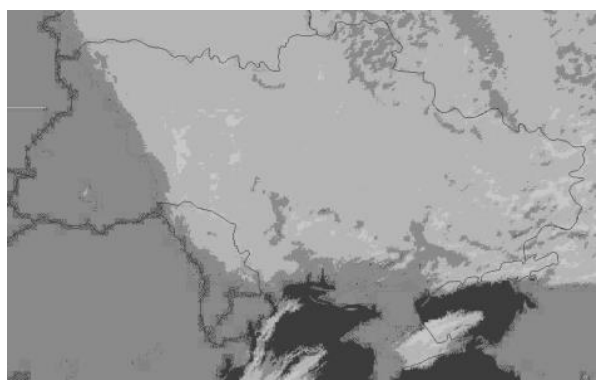

d)

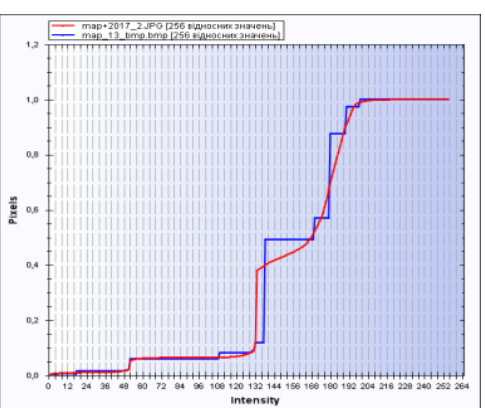

b)

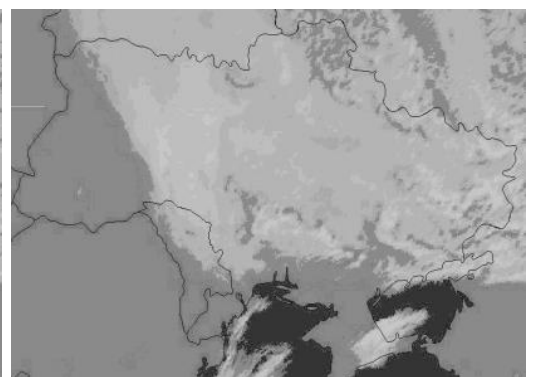

e)

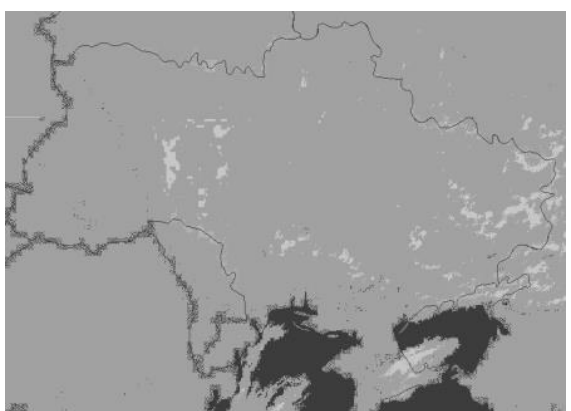

c)

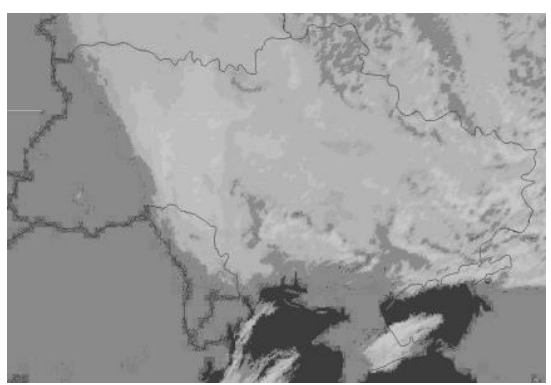

f)

Figure 13 - Results: (a) - satellite map, (b) - cumulative histograms of (a) and (f), (c) - 4 levels segmenting, (d) - 6 levels segmenting, (e) 8 - levels segmenting, (f) - 10 levels segmenting

Table 6. PSNR values for different types of intensity pixel exchange

\begin{tabular}{|c|c|}
\hline Type of intensity assignment & PSNR, dB \\
\hline Left & 30,8544 \\
\hline Right & 30,8439 \\
\hline Center & 31,7404 \\
\hline Weighed & 34,6384 \\
\hline
\end{tabular}

Simultaneously, approximation changes continuous type of images by discrete ones. Approximated images are compressed. Some examples of compression ratios for different format of images are given in Table 7.

Table 7. File dimension and compression ratio

\begin{tabular}{|l|c|c|}
\hline Type of image & JPG & PNG \\
\hline Grayscale & 42,3 & 251,0 \\
\hline Compres Left & 49,6 & 102,7 \\
\hline Ratio Left & 0,85 & 2,44 \\
\hline Compres Right & 48,0 & 103,1 \\
\hline Ratio Right & 0,88 & 2,44 \\
\hline Compres Center & 50,4 & 102,6 \\
\hline Ratio Center & 0,84 & 2,45 \\
\hline Compres Weight & 47,4 & 102,7 \\
\hline Ratio Weight & 0,89 & 2,44 \\
\hline
\end{tabular}

\section{CONCLUSION}

Thus, software of the method of multilevel thresholds for image segmentation based on piecewise linear approximation was developed. The method is based on original RDP algorithm intended to reduce the algorithmic complexity because a cumulative histogram is calculated one time. The developed algorithm was applied to segment cloudiness on the satellite map images. Future investigations are planned to use the approximation algorithm for face and defective images features extraction.

\section{REFERENCES}

[1] N. Otsu, "A threshold selection method from gray level histograms," IEEE Transactions on Systems, Man and Cybernetics, vol. 9, issue 1, pp. 62-66, 1979.

[2] A. Rosenfeld, P. De La Torre, "Histogram concavity analysis as an aid in threshold selection," IEEE Transactions on Systems, Man and Cybernetics, vol. 13, issue 2, pp. 231-235, 1983. 
[3] W. Tsai, "Moment-preserving thresholding: a new approach," Computer Vision, Graphics \& Image Processing, vol. 29, no. 3, pp. 377-393, 1985.

[4] J.N. Kapur, P.K. Sahoo, A.K.C. Wong, "A new method for gray-level picture thresholding using the entropy of the histogram," Computer Vision, Graphics \& Image Processing, vol. 29, no. 3, pp. 273-285, 1985.

[5] P.L. Rosin, L. Paul, "Unimodal thresholding," Pattern Recognition, vol. 34, no. 11, pp. 20832096, 2001.

[6] C.A. Glasbey, "An analysis of histogram-based thresholding algorithms," CVGIP: Graphical Models and Image Processing, vol. 55, issue 6, pp. 532-537, 1993.

[7] P. F. Felzenszwalb, D. P. Huttenlocher, "Efficient graph-based image segmentation," International Journal of Computer Vision, vol. 59, no. 2, pp. 167-181, 2004.

[8] X. Xu, S. Xu, L. Jin, E. Song, "Characteristic analysis of Otsu threshold and its applications," Pattern Recognition Letters, vol. 32, no. 7, pp. 956-961, 2011.

[9] R. Medina-Carnicer, F. Madrid-Cuevas, "Unimodal thresholding for edge detection," Pattern Recognition, vol. 41, no. 7, pp. 23372346, 2008.

[10] P.S. Liao, T.S. Chen, P.C. Chung, "A fast algorithm for multilevel thresholding," Journal of Imformation Science and Engineering, vol. 17, pp. 713-727, 2001.

[11] Y. Huang, S. Wang, "Multilevel thresholding methods for image segmentation with Otsu based on QPSO," Proceedings of the Congress on Image and Signal Processing, 2008, pp. 701-705.

[12] S. Arora, J. Acharya, A. Verma, P.K. Panigrahi, "Multilevel thresholding for image segmentation through a fast statistical recursive algorithm," Pattern Recognition Letters, vol. 29, no. 2, pp. 119-125, 2008.

[13] S.J. Mousavirad, H. Ebrahimpour-Komleh, "Multilevel image thresholding using entropy of histogram and recently developed populationbased metaheuristic algorithms," Evolutionary Intelligence, vol. 10, no. 1-2, pp. 45-75, 2017.

[14] A.Z. Arifin, A. Asano, "Image segmentation by histogram thresholding using hierarchical cluster analysis," Pattern Recognition Letters, vol. 27, no. 13, pp. 1515-1521, 2006.

[15] A.K.M. Khairuzzaman, S. Chaudhury, "Multilevel thresholding using grey wolf optimizer for image segmentation," Expert Systems with Applications, vol. 86, pp. 64-76, 2017.

[16] R. Melnyk, Yu. Kalychak, R. Tushnytskyy, "Absolute and relative classification of cloud regions by satellite image clustering," Proceedings of the IEEE 15th International Conference on the Experience of Designing and
Application of CAD Systems (CADSM), pp. 4549, 2019.

[17] U. Ramer, "An iterative procedure for the polygonal approximation of plane curves," Computer Graphics and Image Processing, vol. 1, issue 3, pp. 244-256, 1972.

[18] D. Douglas, T. Peucker, "Algorithms for the reduction of the number of points required to represent a digitized line or its caricature," The Canadian Cartographer, vol. 10, issue 2, pp. 112-122, 1973.

[19] R. Melnyk, Yu. Kalychak, "Image thresholding by extrema coordinates of cumulative histogram," International Journal Sensing, Computing \& Control IJSCC, vol. 4, issue 1, pp. 1-9, 2014.

[20] Infrared satellite images of Europe, Clouds in Europe at night and in the evening. Weather Europe, Satellite Weather Europe, SAT24.com, [Online]. Available at: https://es.sat24.com/en.

[21] Satellite images of Ukraine, [Online]. Available at: https://meteo.gov.ua/33345/satellite.

[22] Precipitation in Finland, [Online]. Available at: https://en.ilmatieteenlaitos.fi/rain-and-cloudiness.

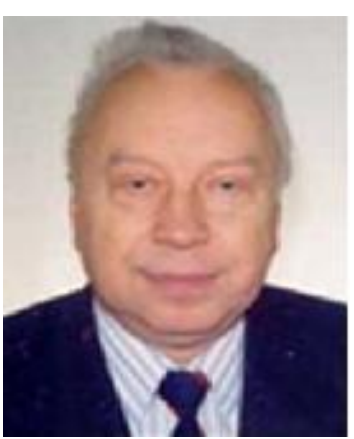

Roman Melnyk, a Professor of Software Engineering Department, Institute of Computer Sciences and Information Technologies, Lviv Polytechnic National University. Scientific interests: fuzzy clustering, recognition of visual information

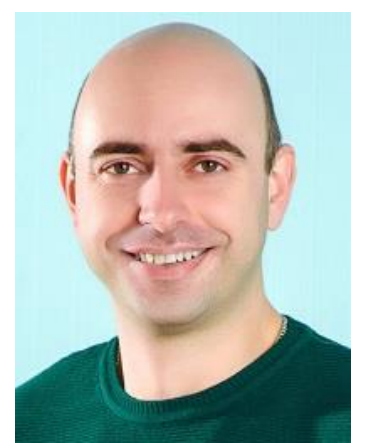

Ruslan Tushnytskyy, an Associate Professor of Software Engineering Department, Institute of Computer Sciences and Information Technologies, Lviv Polytechnic National University. Scientific interests: image analysis and processing, pattern recognition.

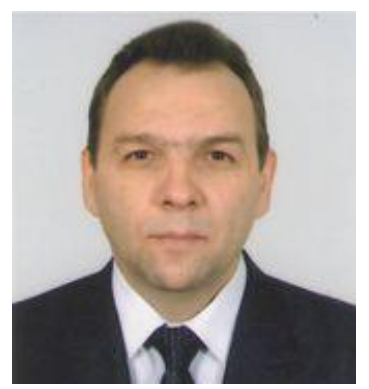

Roman Kvit, an Associate Professor of Mathematics Department, Institute of Applied Mathematics and Fundamental Sciences, Lviv Polytechnic National University. Scientific interests: numerical and mathematical modeling. 